\author{
J. Beier ${ }^{1}$ \\ K. M. Beeh ${ }^{1}$ \\ K. Tröger ${ }^{3}$ \\ S. Stenglein ${ }^{3}$ \\ M. Bräutigam ${ }^{1}$ \\ R. Buhl ${ }^{1}$ \\ E. W. Schmidt ${ }^{2}$
}

\section{Bodyplethysmographischer Wirkeintritt von Formoterol bei Patienten mit mittel- bis schwer- gradiger partiell reversibler Atemwegsobstruktion}

Onset of Action of Formoterol in Patients with Moderate to Severe, Partially Reversible Airflow Obstruction Assessed by Bodyplethysmography

\section{Zusammenfassung}

Hintergrund: Formoterol ist ein langwirksames, inhalatives $\beta_{2}$-Sympathomimetikum zur Behandlung obstruktiver Atemwegserkrankungen. Darüber hinaus zeichnet sich Formoterol gegenüber Salmeterol durch einen schnelleren Wirkeintritt aus. Bislang liegen nur wenige vergleichende Untersuchungen zum bodyplethysmographischen Wirkeintritt von Formoterol und Salmeterol bei mittel- bis schwergradiger, partiell reversibler Atemwegsobstruktion vor. Methode: In einer monozentrischen, einfach-blinden Parallelgruppen-Studie wurde der Wirkeintritt von Formoterol $\left(2 \times 12 \mu \mathrm{g} /\right.$ Tag via Foradil Aerolizer $\left.{ }^{\circledR}\right)$ und Salmeterol $\left(2 \times 50 \mu \mathrm{g} /\right.$ Tag via Serevent Diskus $\left.{ }^{\circledR}\right)$ vor und nach einer 14-tägigen Behandlung bodyplethysmographisch bei 60 Patienten mit mittel- bis schwergradiger Atemwegsobstruktion (Alter: 61,9 $\pm 12,8$ Jahre, 65\% männlich, FEV $_{1} 40,6 \pm 15,3 \%$ des Sollwertes; Reversibilität $>15 \%$ ) untersucht. Ergebnisse: Nach Inhalation von Formoterol zeigte sich sowohl initial (Tag 1) als auch nach 14-tägiger Behandlung ein signifikant schnellerer Wirkungseintritt (Reduktion von sRAw um $>10 \%$ ) als in der Salmeterolgruppe (Visite 1: 1,4 $\pm 0,9$ vs. 15,1 $\pm 34,5$ min; Visite 3: $6,2 \pm$ 21,6 vs. $51 \pm 135$ min., $\mathrm{p}<0,0001$, beide Vergleiche). In beiden Gruppen konnte nach 14-tägiger Behandlung eine deutliche Verbesserung der morgendlichen Lungenfunktion $\left(\mathrm{FEV}_{1}\right)$ gegenüber dem Ausgangswert beobachtet werden (Formoterol: 1,38 $\pm 0,641$ vs. $1 \pm 0,41$; Salmeterol: $1,43 \pm 0,671$ vs. $1 \pm 0,4, p<0,05$, beide Vergleiche). Sowohl Formoterol als auch Salmeterol waren gut verträglich. Zusammenfassung: Auch bei Patienten mit mittelbis schwergradiger Atemwegsobstruktion lässt sich bodyplethysmographisch nach Inhalation von Formoterol aus dem Aero-

\section{Abstract}

Background: Formoterol is a long acting $\beta_{2}$-agonist used for the treatment of obstructive airway diseases. Compared with Salmeterol, Formoterol has a rapid onset of bronchodilation. There are only scant data regarding the comparative onset of action using bodyplethysmography in moderate to severely obstructive patients. Methods: In a mono-center, single-blinded parallel group study 60 patients (age: $61.9 \pm 12.8$ years, $65 \%$ male) with moderate to severe (mean $\mathrm{FEV}_{1} 40.6 \pm 15.3 \%$ of predicted), partially reversible $\left(\mathrm{FEV}_{1}\right.$ post bronchodilator $>15 \%$ from baseline) airway obstruction were treated with either formoterol $12 \mu \mathrm{g}$ bid or salmeterol $50 \mu \mathrm{g}$ bid over a period of two weeks. Onset of action was measured by airway resistance (sRaw) before and after two weeks of treatment. Results: Compared with Salmeterol, Formoterol had a significantly faster onset of action (10\% decrease of Raw) at baseline (F: $1.4 \pm 0.9$ vs. S: $15.1 \pm 34.5$ min., p<0.0001) and after two weeks of treatment (F: $6.2 \pm 21.6$ vs. S: $51 \pm$ 135 min., $\mathrm{p}<0.0001$ ). Morning $\mathrm{FEV}_{1}$ improved similarily during treatment in both study groups, when compared with baseline lung function (F: $1.38 \pm 0.64$ vs. $1 \pm 0.41 \mathrm{l}$; S: $1.43 \pm 0.67$ vs. $1 \pm$ $0.41, \mathrm{p}<0.05$, both comparisons). Both treatments were well tolerated. Conclusion: Formoterol produces a rapid improvement of airway resistance in patients with moderate to severe, partially reversible airway obstruction. The onset of bronchodilation was significantly faster for Formoterol compared with Salmeterol. Both drugs improved lung function similarily after two weeks of treatment. It is important to distinguish $\beta_{2}$-agonists not only into short- and long-acting but also into fast- and slowacting. 
lizer ${ }^{\circledR}$ eine rasche Bronchodilatation erzielen. Dabei war Formoterol Salmeterol bezüglich der Geschwindigkeit des Wirkungseintritts überlegen. Die mit beiden Substanzen erzielte Kontrolle der obstruktiven Ventilationsstörung war dagegen vergleichbar. Neben der Unterscheidung lang- und kurzwirksamer $\beta_{2}$-Sympathomimetika erscheint eine Klassifizierung in schnell und verzögert wirksame $\beta_{2}$-Sympathomimetika sinnvoll.

\section{Hintergrund}

Formoterol und Salmeterol sind langwirksame $\beta_{2}$-Sympathomimetika mit einer dokumentierten Wirkdauer von über 10-12 Stunden. Gegenüber Salmeterol ist Formoterol ein voller Agonist am $\beta_{2}$-Rezeptor mit höherer intrinsischer Aktivität. Formoterol zeigt einen schnelleren Wirkungseintritt als Salmeterol sowohl in vitro an der glatten Muskelzelle $[1,2]$ als auch in vivo bei asthmatischen Patienten [3,4]. Anders als Salmeterol kann Formoterol daher auch zur Kupierung einer akuten Atemwegsobstruktion eingesetzt werden. Aktuelle Untersuchungen deuten darauf hin, dass die bedarfsadaptierte Inhalation von Formoterol gegenüber kurzwirksamen $\beta_{2}$-Sympathomimetika wie Terbutalin bei gleicher Verträglichkeit überlegen ist [5]. Der Stellenwert von Formoterol und Salmeterol in der Therapie obstruktiver Atemwegserkrankungen ist durch eine Vielzahl klinischer Studien belegt $[6,7]$. Sind z. B. asthmatische Beschwerden trotz einer konsequenten Therapie mit topischen Kortikoiden nicht zu kontrollieren und besteht ein gehäufter Bedarf an kurzwirksamen $\beta_{2}$-Sympathomimetika mit insbesondere nächtlichen Beschwerden, so sehen aktuelle Therapieempfehlungen eine Erweiterung der Behandlung um ein langwirksames Bronchospasmolytikum vor [8]. Es ist wiederholt gezeigt worden, dass bei mangelhafter Asthma-Kontrolle die zusätzliche Gabe langwirksamer inhalativer $\beta_{2}$-Sympathomimetika einer Erhöhung der Dosis topischer Steroide hinsichtlich der klinischen Wirksamkeit überlegen ist $[6,7,9]$. Gegenüber einer Dauertherapie mit kurzwirksamen $\beta_{2}$-Sympathomimetika verbessern langwirksame $\beta_{2}$-Sympathomimetika alle funktionellen Parameter der obstruktiven Ventilationsstörung, die klinische Symptomatik, Nachtruhe und Lebensqualität von Patienten mit Asthma bronchiale bei vergleichbarer Verträglichkeit [10-12].

Auch für die chronisch obstruktive Lungenerkrankung (chronic obstructive pulmonary disease, COPD) liegen mittlerweile zahlreiche Untersuchungen zur Wirksamkeit langwirksamer $\beta_{2}$-Sympathomimetika vor [13,14]. Zwischen Asthma bronchiale und COPD bestehen klinisch zahlreiche Überlappungen, die eine diagnostische Unterscheidung in der klinischen Praxis mit realistischem Aufwand häufig schwierig machen. Wenngleich es sich bei der COPD definitionsgemäß um eine Erkrankung mit allenfalls geringgradig reversibler Atemwegsobstruktion handelt [15], weisen viele Patienten dennoch eine partielle Reversibilität in der Lungenfunktionsprüfung auf $[16,17]$. Dabei ist wiederholt gezeigt worden, dass die alleinige Erfassung spirometrischer Parameter wie 1-Sekunden-Kapazität $\left(\mathrm{FEV}_{1}\right)$ nicht ausreichend repräsentativ für die Beurteilung des Effektes einer bronchospasmolytischen Substanz ist. Vielmehr scheinen hier andere physiologische Messgrößen wie pulmonale Überblähung, 6-MinutenLauftest oder FIV ${ }_{1}$ zum Teil empfindlichere Zielgrößen zu sein
$[18,19]$. Mit der Bodyplethysmographie lassen sich über die Spirometrie hinaus Lungenfunktionsgrößen wie spezifischer Atemwegswiderstand (sRaw) zuverlässig und reproduzierbar erfassen. Bislang liegen zu bodyplethysmographischen Effekten von langwirksamen $\beta_{2}$-Sympathomimetika bei mittel- bis schwergradiger Atemwegsobstruktion nur vereinzelte Untersuchungen vor $[20,21]$. Ziel der vorliegenden Untersuchung war daher die Überprüfung des Wirkeintritts von Formoterol bei Patienten mit mittel- bis schwergradiger, partiell reversibler obstruktiver Atemwegserkrankung. Bei diesen Patienten erscheint beim Gebrauch von $\beta_{2}$-Sympathomimetika sowohl in der Dauertherapie als auch bei akuten Beschwerden ein rascher Wirkungseintritt wünschenswert. Es wurde daher als primäres Zielkriterium prospektiv die Zeit bis zum Eintritt einer bronchodilatatorischen Wirkung vor und nach einer 14-tägigen Behandlung mit Formoterol (Pulverinhalation mittels Aerolizer ${ }^{\circledR}$ ) oder Salmeterol (Pulverinhalation mittels Diskus ${ }^{\circledR}$ ) untersucht.

\section{Material und Methoden}

\section{Patienten}

Es wurden 60 Patienten mit mittel- bis schwergradiger Atemwegsobstruktion (IVC 2,371 $\pm 0,681$, Spanne 1,06-3,881; $\mathrm{FEV}_{1}$ $1,091 \pm 0,461[40,6 \pm 15 \%$ des Sollwertes], Spanne 0,45 l-2,311 [17-73\% des Sollwertes]; sRaw 5,7 $\pm 3,31 \mathrm{kPa}^{*} \mathrm{~s}$, Spanne 2-17 kPa*s) nach ATS-Kriterien [22] in die Studie eingeschlossen. Einschlusskriterien waren neben der dokumentierten Atemwegsobstruktion eine partielle Reversibilität der obstruktiven Atemwegserkrankung (> 15\% des Ausgangswertes) nach Inhalation von $400 \mu g$ Salbutamol (Glaxo Wellcome, Bad Oldesloe). Die Therapie der obstruktiven Atemwegserkrankung sowie etwaiger Begleiterkrankungen musste in den letzten 4 Wochen vor Studienbeginn unverändert geblieben sein, insbesondere galt dies für die Dosis inhalierbarer und/oder systemischer Kortikosteroide. Die bestehende Medikation der obstruktiven Atemwegserkrankung wurde unverändert fortgesetzt, mit Ausnahme oraler oder inhalativer langwirksamer $\beta_{2}$-Sympathomimetika während der Studie.

Ausschlusskriterien waren eine Exazerbation der Atemwegserkrankung und/oder eine akute Infektion der Atemwege, maligne Erkrankungen, Schwangerschaft bzw. Stillzeit, fehlende Kontrazeption, pathologische Laborabweichungen in Blutbild und/oder klinischer Chemie (Leber- und Nierenparameter), eine nach Urteil des Prüfarztes mit der Teilnahme an der Prüfung unvereinbare schwerwiegende Grund- oder Begleiterkrankung sowie die Teilnahme an einer anderen klinischen Prüfung während 30 Tagen vor Studienbeginn.

\section{Studiendesign}

Die randomisierte, kontrollierte, prospektive, einfach-blinde Parallelgruppen-Studie wurde monozentrisch zwischen Dezember 1997 und August 1999 am Klinikum Chemnitz durchgeführt.

Die Prüfmedikation bestand aus Formoterol (Foradil Aerolizer ${ }^{\circledR}$, $12 \mu \mathrm{g} /$ Inhalationskapsel, Tagesdosis $2 \times 12 \mu \mathrm{g}$; Novartis Pharma AG, Basel, Schweiz) oder Salmeterol (Serevent Diskus ${ }^{\circledR}$, $50 \mu \mathrm{g} / \mathrm{Hub}$, Tagesdosis $2 \times 50 \mu \mathrm{g}$; Glaxo Wellcome GmbH, Bad Oldesloe) und wurde morgens und abends im Abstand von 12 Stunden inhaliert. Zur Kontrolle akuter obstruktiver Beschwer- 
den erhielten die Patienten ein kurzwirksames $\beta_{2}$-Sympathomimetikum (Salbutamol Dosieraerosol, $100 \mu \mathrm{g} / \mathrm{Hub}$, Glaxo Wellcome GmbH, Bad Oldesloe).

Die Studiendauer betrug 15 Tage. Es erfolgten insgesamt 4 Visiten (Screening, Visite 1: Tag 1, Visite 2: Tag 7, Visite 3: Tag 14) pro Patient. Untersuchungen hinsichtlich des Wirkeintritts der Prüfsubstanzen wurden bei Visite 1 und bei Visite 3 durchgeführt.

Während der gesamten Studiendauer wurde die Medikamenteneinnahme zur Überprüfung der Compliance in Tagebuchform festgehalten. Körperliche Untersuchungen und Laborkontrollen inklusive klinischer Chemie, Glukose und Serum-Elektrolyte erfolgten beim Screening sowie den Visiten 2 und 3.

Prüfplan und Patienteninformationen wurden von der Ethikkommission der Sächsischen Ärztekammer geprüft und genehmigt. Die Studie wurde unter Beachtung aktueller Richtlinien (Arzneimittelgesetz, Good Clinical Practice, Deklaration von Helsinki) durchgeführt. Alle Studienteilnehmer waren bei Studieneintritt volljährig und erklärten nach eingehender mündlicher und schriftlicher Aufklärung über Zweck und Durchführung der Medikamentenprüfung schriftlich ihr Einverständnis.

\section{Lungenfunktion (Bodyplethysmographie, Spirometrie)}

Primäres Zielkriterium war die Zeit bis zum Wirkeintritt von Formoterol und Salmeterol an Tag 1 und nach 14-tägiger Behandlungsdauer. Als Zeitpunkt des Wirkeintrittes wurde der Zeitpunkt eines 10\%igen Abfalls des spezifischen Atemwegswiderstandes (sRAw) gegenüber dem Ausgangswert definiert, da die Variabilität der Methode unter standardisierten Bedingungen zwischen 5 und $10 \%$ beziffert wird $[23,24]$. Der sRaw wurde bodyplethysmographisch an Tag 1 und 14 in der ersten Stunde nach Inhalation der Prüfsubstanz zu insgesamt 10 Zeitpunkten $(0,1,2$, $3,5,10,15,20,30,60$ Minuten) sowie im weiteren Verlauf stündlich bis Stunde 12 gemessen.

Zusätzlich zur Bestimmung von sRaw wurde beim Screening sowie am Morgen von Visite 1 und 3 die Ein-Sekundenkapazität $\left(\mathrm{FEV}_{1}\right)$ bestimmt.

Vor den Messungen durften mindestens 12 Stunden keine langwirksamen, bzw. 4 Stunden keine kurzwirksamen $\beta_{2}$-Sympathomimetika appliziert werden.

Die Messungen wurden in einem Ganzkörperplethysmographen (Jäger, Masterlab II, Würzburg) mit integriertem Spirometriemessplatz nach den Qualitätskriterien der ATS durchgeführt.

\section{Verträglichkeit}

Während des gesamten Prüfzeitraumes wurden vom Prüfarzt unerwünschte Ereignisse (UE) und Begleitmedikation erfasst. Jedes im Studienzeitraum aufgetretene unerwünschte Ereignis wurde unabhängig von einem möglichen Zusammenhang mit der Prüfmedikation dokumentiert. Unerwünschte Ereignisse waren alle beim Patienten auftretenden Befindlichkeitsstörungen bzw. subjektiven oder objektiven Krankheitssymptome. Als schwerwiegend wurden unerwünschte Ereignisse eingestuft, wenn sie lebensbedrohlich waren bzw. zu einem stationären Krankenhausaufenthalt, einer dauernden und/oder schweren
Behinderung führten, sowie jeder Todesfall und jede maligne Erkrankung. Darüber hinaus erfolgte bei jedem Arztbesuch eine körperliche Untersuchung mit Dokumentation von Blutdruck und Puls.

\section{Statistische Analyse}

Alle Resultate sind, soweit nicht anders angeführt, als arithmetische Mittelwerte \pm Standardabweichung (SD) bzw. als Median und Spanne angegeben. Die Analyse der Zeit bis zum Wirkeintritt (sRaw $-10 \%$ prä Inhalationem ) wurde mittels Rangsummentest oder Vorzeichentest nach Wilcoxon vorgenommen. Es wurde dabei das Intention-to-treat-Prinzip (ITT) zugrunde gelegt, d.h. jeder Patient, der mindestens einmal Formoterol oder Salmeterol angewendet hatte, wurde in die Analyse einbezogen $(n=60)$. Fehlende Werte von Zwischenkontrollen wurden nach dem Prinzip „Last Value Carried Forward“ ergänzt. Das Signifikanzniveau wurde bei einer Irrtumswahrscheinlichkeit von unter 5\% $(p<0,05)$ angesetzt.

\section{Ergebnisse}

\section{Patienten}

Das ITT-Kollektiv bestand aus 60 Patienten, die randomisiert einem der beiden Behandlungsarme zugeteilt wurden (30 Patienten Formoterol $2 \times 12 \mu \mathrm{g}$, 30 Patienten Salmeterol $2 \times 50 \mu \mathrm{g}$ ). Die demografischen Daten waren in der Formoterolgruppe und der Salmeterolgruppe vergleichbar. Ebenso unterschieden sich Dauer und Schwere der obstruktiven Atemwegserkrankung in beiden Gruppen nicht (Tab.1). 21 Patienten (70\%) in der Formoterol- und 20 Patienten (66,7\%) in der Salmeterolgruppe standen im Studienzeitraum unter einer systemischen Kortikosteroidtherapie.

Tab. 1 Demografische Daten bei Visite 1

\begin{tabular}{|lll}
\hline & $\begin{array}{l}\text { Salmeterol } \\
\text { Diskus }\end{array}$ & $\begin{array}{l}\text { Formoterol } \\
\text { Aerolizer }\end{array}$ \\
\hline Patientenzahl (n) & 30 & 30 \\
\hline Alter (Jahre: Median/Spanne) & $66(40-78)$ & $63,5(28-84)$ \\
\hline Geschlecht (M\% : W\%) & $70: 30$ & $60: 40$ \\
\hline BMI (kg/m²) & $26 \pm 4,2$ & $26 \pm 5,1$ \\
\hline KH-Dauer (Jahre: Median/Spanne) & $11,5(1-59)$ & $9,5(1-53)$ \\
\hline FEV ${ }_{1}$ (\% Sollwert, Mittelwert) & $40,7 \pm 17,6$ & $40,5 \pm 13,3$ \\
\hline
\end{tabular}

Der Anteil aktiver, bzw. ehemaliger Raucher war mit 21,7\% bzw. $55 \%$ hoch. Aufgrund der Anamnese (Alter, Rauchgewohnheiten, Allergien) hatten $76,6 \%$ der Patienten nach Einschätzung der Prüfärzte eine COPD (ICD 10 J 44.9), bei $23,4 \%$ der Patienten lag ein Asthma bronchiale (ICD J45.0 und J 45.1) vor. Die prozentuale Verteilung der Rauchgewohnheiten und beider Diagnosen war in den Behandlungsarmen jeweils vergleichbar.

Häufige Begleiterkrankungen waren Herzinsuffizienz (32\%), Adipositas (28,3\%), essenzielle Hypertonie (25\%), Diabetes mellitus (15\%) und Hyperurikämie (13\%). Atopische Begleiterkrankungen lagen nur bei wenigen Studienteilnehmern vor (allergische Rhinitis: $8 \%$, atopische Dermatitis: 6,7\%). 
Visite 1

\section{Wirkeintritt (spezifischer Atemwegswiderstand sRaw)}

Der morgendliche mittlere spezifische Atemwegswiderstand vor der Inhalation der Studienmedikation war bei Visite 1 in beiden Gruppen vergleichbar (Formoterol: 5,49 $\pm 3,32 \mathrm{kPa}$ s; Salmeterol: 5,92 $\left.\pm 3,35 \mathrm{kPa}^{*} \mathrm{~s} ; \mathrm{p}>0,1\right)$. Nach erster Inhalation der Prüfsubstanzen kam es in beiden Therapiearmen zu einem Abfall des sRaw auf im Mittel maximal 43,7\% (Formoterol) bzw. 49,3\% (Salmeterol) des Ausgangswertes. Bezüglich der maximalen Wirkung auf sRaw fanden sich zwischen Formoterol und Salmeterol keine signifikanten Unterschiede. In der Formoterolgruppe kam es jedoch signifikant früher als in der Salmeterolgruppe zum definierten Wirkeintritt (1,43 $\pm 0,9$ vs. $15,13 \pm 34,55$ Minuten nach Inhalation; $p<0,0001 ;$ Abb. 1).

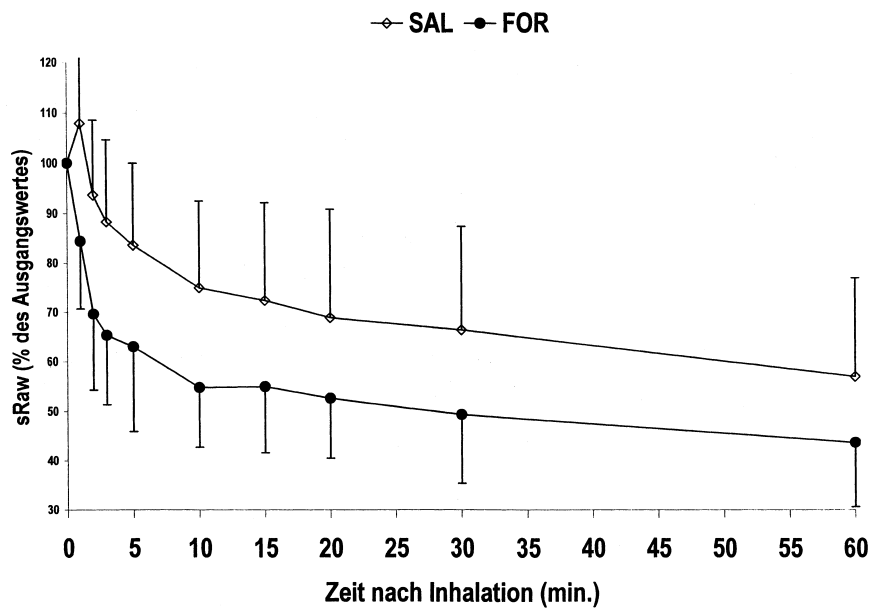

Abb. 1 Wirkeintritt (sRaw - 10\%) von Formoterol und Salmeterol bei Visite 1. (Mittelwert sRaw (\% des Ausgangswertes) und Standardabweichung).

\section{Visite 3}

\section{Wirkeintritt (spezifischer Atemwegswiderstand sRaw)}

Nach zwei Wochen Behandlung mit Formoterol oder Salmeterol fand sich in beiden Gruppen ein gegenüber dem Ausgangswert (vor Behandlung) signifikant verminderter sRaw (Formoterol $5,49 \pm 3,32 \mathrm{kPa}^{*} \mathrm{~s}$ vs. $3,47 \pm 2,14 \mathrm{kPa}^{*} \mathrm{~s} ; \quad$ Salmeterol 5,92 \pm $3,35 \mathrm{kPa}$ *s vs. 4,18 $\pm 3,8 \mathrm{kPa}{ }^{*} \mathrm{~s} ; \mathrm{p}<0,05$; beide Vergleiche). Korrespondierend hierzu fand sich in beiden Gruppen ein verbessertes morgendliches $\mathrm{FEV}_{1}$ (Abb.3). Zwischen den Behandlungsgruppen fanden sich bei Visite 3 für den morgendlichen mittleren Atemwegswiderstand vor Inhalation der Studienmedikation keine signifikanten Unterschiede (Formoterol: 3,47 $\pm 2,14 \mathrm{kPa}^{*} \mathrm{~s}$; Salmeterol: 4,18 $\pm 3,8 \mathrm{kPa} *$; $\mathrm{p}>0,1)$.

Nach Inhalation der Prüfmedikation kam es in der Formoterolgruppe erneut signifikant früher als in der Salmeterolgruppe zum definierten Wirkeintritt $(6,23 \pm 21,61 \mathrm{~min}$. vs. 51,03 \pm 135,21 min. nach Inhalation; $p<0,0001$ ) (Abb. 2). Bezüglich des maximalen Effektes gab es auch bei Visite 3 keine Unterschiede zwischen der Formoterol- bzw. Salmeterolgruppe.

\section{Verträglichkeit}

Im Verlauf der Studie trat ein schwerwiegendes unerwünschtes Ereignis (eitrige Bronchitis mit verlängerter Hospitalisierung)

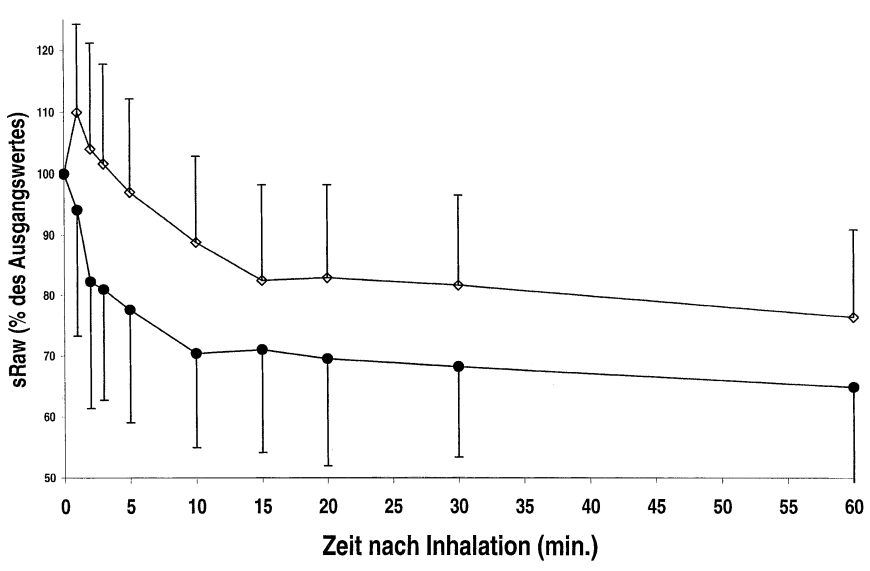

Abb. 2 Wirkeintritt (sRaw - 10\%) von Formoterol und Salmeterol bei Visite 3 (Mittelwert sRaw (\% des Ausgangswertes) und Standardabweichung).

\section{Visite $1 \square$ Visite 3}

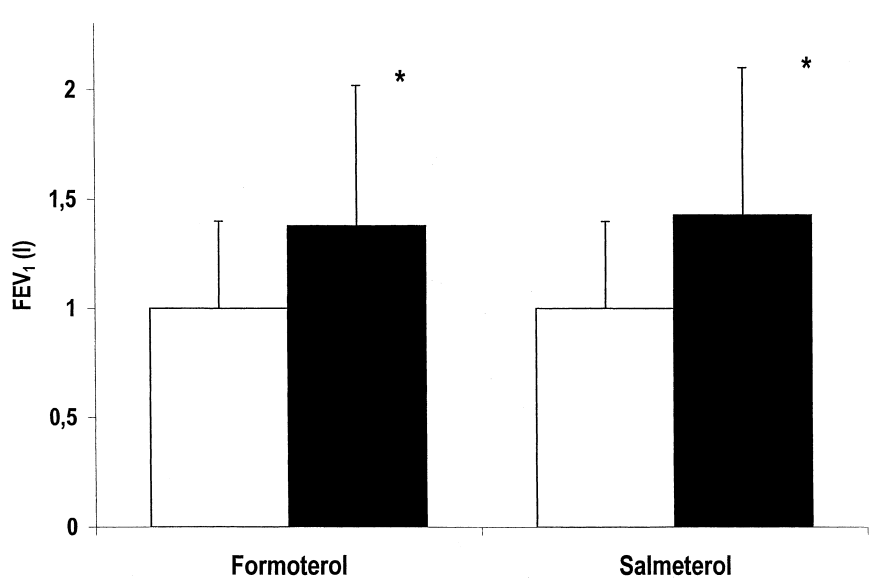

Abb. 3 Ausgangswerte für das $\mathrm{FEV}_{1}$ bei Visite 1 und nach 14-tägiger Behandlung bei Visite 3 in beiden Gruppen (Mittelwert FEV F $_{1} \mathrm{l}$ ) und Standardabweichung; ${ }^{*} p<0,05$, beide Vergleiche).

auf, das jedoch vom behandelnden Prüfarzt nicht mit der Prüfmedikation (Formoterol) in Zusammenhang gebracht wurde.

Desweiteren kam es im Verlauf des Beobachtungszeitraumes zu 9 nicht schwerwiegenden unerwünschten Ereignissen (UE), deren Zusammenhang mit der Prüfmedikation vom Prüfarzt als „möglich“, „wahrscheinlich“ oder „fast sicher“ angenommen wurde. Diese UE betrafen Symptome der bekannten Nebenwirkungen von $\beta_{2}$-Sympathomimetika (Kopfschmerzen, Tremor, Schwindel und Schlafstörungen) und Sodbrennen (Tab. 2).

Blutdruck und Puls waren in beiden Behandlungsgruppen gleich und blieben während der Behandlungsphase unverändert. Laborwerte (Hämatologie, klinische Chemie, Elektrolyte) blieben im Mittel im Normbereich, es traten keine klinisch relevanten Veränderungen der Laborwerte auf, die auf die Studienmedikation zurückzuführen waren. Bei einem Patienten aus der Formoterolgruppe kam es unter der Therapie zu einer milden Hypokaliämie $\left(\mathrm{K}^{+} 2,9 \mathrm{mmol} / \mathrm{l}\right)$. 
Tab. 2 Unerwünschte Ereignisse mit nach Einschätzung des Prüfarztes möglichem, wahrscheinlichem oder sicherem Zusammenhang mit der Prüfmedikation

\begin{tabular}{lll}
\hline $\begin{array}{l}\text { unerwünschtes } \\
\text { Ereignis }\end{array}$ & Formoterol & Salmeterol \\
\hline Kopfschmerzen & 1 & 2 \\
\hline $\begin{array}{l}\text { Tremor } \\
\text { Sodbrennen }\end{array}$ & 1 & \\
\hline $\begin{array}{l}\text { Schwindel } \\
\text { Schlafstörungen }\end{array}$ & 2 & 2 \\
\hline
\end{tabular}

Diskussion

Ziel dieser prospektiven, einfach-blinden, monozentrischen Studie war der bodyplethysmographische Vergleich des Wirkeintritts der beiden langwirksamen $\beta_{2}$-Sympathomimetika Formoterol und Salmeterol vor und nach einer 14-tägigen Behandlung bei Patienten mit mittel- bis schwergradiger, partiell reversibler Atemwegsobstruktion. Die Ergebnisse belegen den gegenüber Salmeterol auch bodyplethysmographisch dokumentierten schnelleren Wirkeintritt von Formoterol, sowohl initial als auch nach Abschluss einer 14-tägigen Dauertherapie mit Formoterol bzw. Salmeterol. Beide Substanzen unterschieden sich im maximalen Wirkeffekt nicht signifikant.

In die klinische Prüfung wurden Patienten mit mittel- bis schwergradiger obstruktiver Atemwegserkrankung aufgenommen. Das mittlere $\mathrm{FEV}_{1}$ lag bei allen Patienten etwa um $40 \%$ des Sollwertes. Für diese Patienten sehen alle aktuellen Therapieempfehlungen von Fachgesellschaften eine Dauertherapie mit langwirksamen $\beta_{2}$-Sympathomimetika vor unabhängig davon, ob es sich um eine eher als Asthma oder eher als COPD anzusprechende Erkrankung handelt. Bei der Auswahl der Teilnehmer wurde daher auf eine strenge Trennung zwischen Patienten mit Asthma bronchiale und COPD verzichtet, zumal diese Differenzierung im klinischen Alltag mit realistischem Aufwand häufig ohnehin nicht durchführbar ist. Die für ein Kollektiv von Patienten mit obstruktiven Atemwegserkrankungen niedrige Prävalenz atopischer Begleiterkrankungen, der hohe Anteil aktiver und ehemaliger Raucher sowie Altersverteilung und Spektrum der Begleiterkrankungen legen jedoch nahe, dass es sich bei der überwiegenden Mehrzahl der Probanden um Patienten mit COPD handelte. Gerade diese Patienten stellen im klinischen Alltag häufig Problemfälle dar, die zudem eine breite und relevante Ko-Morbidität aufweisen. Trotz der offensichtlichen Dominanz von COPD-Patienten in dem vorgestellten Patientenkollektiv wiesen alle Probanden eine Reversibilität der Atemwegsobstruktion, gemessen am $\mathrm{FEV}_{1}$, von mindestens $15 \%$ auf. Anthonisen $\mathrm{u}$. Mitarb. untersuchten die Reversibilität von 985 Patienten mit COPD, und fanden eine partielle Reversibilität von mindestens $20 \%$ bei einem knappen Drittel der Patienten [16]. Auch andere Untersuchungen weisen darauf hin, dass bei einem substanziellen Anteil von COPD-Patienten keine fixe, irreversible Bronchialobstruktion vorliegt [17,25]. Es ist wiederholt darüber diskutiert worden, inwieweit es sich bei solchen Patienten um eine „echte“ COPD handelt [26]. Dennoch bleibt festzuhalten, dass in der täglichen ärztlichen Praxis eine exakte Differenzierung häufig nicht getroffen wird $[27,28]$ bzw. die Indikation für eine Therapie mit langwirksamen $\beta_{2}$-Sympathomimetika sich häufig am unmittelbaren klinischen Effekt orientiert.

In der vorliegenden Untersuchung wurde primär der Effekt von Formoterol und Salmeterol auf bodyplethysmographische Zielgrößen untersucht. Bislang liegen zu der hier vorgestellten Patientengruppe mit mittel- bis schwergradiger, reversibler Atemwegsobstruktion keine Daten zu bodyplethysmographischen Effekten vor. Die Mehrzahl der Untersuchungen mit Bodyplethysmographie wurde mit pädiatrischen Patienten durchgeführt. Gegenüber der spirometrischen Erfassung von $\mathrm{FEV}_{1}$ konnten Morice u. Mitarb. zeigen, dass die bodyplethysmographische Bestimmung der spezifischen Leitfähigkeit (sGaw) sensitiver und weniger variabel den Effekt einer bronchodilatatorischen Substanz widerspiegelte [20]. Diese Arbeiten belegen den ergänzenden Stellenwert der Bodyplethysmographie, insbesondere im Hinblick auf die generelle Wertigkeit der Zielgröße $\mathrm{FEV}_{1}$ bei Patienten mit COPD.

Nach Inhalation von Formoterol kam es innerhalb der ersten Minuten zu einem raschen Abfall des sRaw. Der im Studienprotokoll definierte Wirkeintritt (Abfall des sRaw um 10\% des Ausgangswertes) wurde dabei im Mittel bereits 1,43 Minuten nach Inhalation erreicht. Demgegenüber konnte der gleiche Effekt in der Salmeterolgruppe erst nach 15,13 Minuten beobachtet werden. Diese Ergebnisse sind vergleichbar mit spirometrischen Daten einer Dosis-Wirkungs-Studie von Cazzola u. Mitarb. Allerdings wurden dabei Formoterol bzw. Salmeterol via Dosieraerosole appliziert [29]. Auch nach 14 Tagen Dauertherapie mit Formoterol bzw. Salmeterol ließ sich dieser Unterschied belegen (Wirkeintritt nach 6,23 (Formoterol) vs. 51,03 Minuten (Salmeterol), $p=0,001$ ). Auffällig war, dass dabei der Wirkeintritt für beide Substanzen nach 14 Tagen gegenüber dem Ausgangswert verzögert zu verzeichnen war. Dies ist möglicherweise im Rahmen der deutlich verbesserten Ausgangslungenfunktion in beiden Patientengruppen zu interpretieren, da aufgrund des gewählten Zielkriteriums einer 10\%igen Verbesserung von sRaw gegenüber dem Ausgangswert (vor Inhalation) diese Steigerung bei einigen Patienten erst unter der maximalen Wirkung der jeweiligen Substanz erreicht wurde. Dabei ließ sich der maximale Effekt von Formoterol bzw. Salmeterol auf sRaw sowohl bei Visite 1 als auch bei Visite 3 zwischen 60 und 120 Minuten bzw. 120-180 Minuten dokumentieren. Gleichwohl kann die Entwicklung einer zusätzlichen Toleranz gegenüber dem bronchodilatatorischen Effekt beider Substanzen nicht völlig ausgeschlossen werden. Allerdings war das vorliegende Studiendesign nicht dazu geeignet, eine eventuelle Toleranzentwicklung bezüglich bronchoprotektiver bzw. bronchodilatatorischer Effekte von langwirksamen $\beta_{2}$-Sympathomimetika zu erfassen. Weder wurde die unspezifische bronchiale Hyperreagibilität erfasst, noch war die Behandlungsdauer ausreichend lang zur Beantwortung dieser Fragestellung. Dennoch muss man aufgrund der vorliegenden Daten die Entwicklung von Toleranzeffekten bei der Therapie mit langwirksamen $\beta_{2}$-Sympathomimetika sowohl in der Kurz- als auch Langzeittherapie in Betracht ziehen und gegebenenfalls mit einem geeigneten Studiendesign überprüfen $[30,31]$. In diesem Zusammenhang sollte erwähnt werden, dass alle untersuchten Patienten entweder mit inhalativen oder systemischen Kortikosteroiden behandelt worden waren. 
Insgesamt belegen und ergänzen die vorliegenden Daten andere Arbeiten zum raschen Wirkeintritt von Formoterol und legen nahe, dass die Substanz auch bei Patienten mit schwergradiger Atemwegsobstruktion als Notfallmedikament geeignet sein könnte. Cazzola u. Mitarb. untersuchten die Wirksamkeit von Formoterol als Bedarfsmedikation bei COPD-Patienten mit einer akuten Exazerbation [32]. Dabei zeigte Formoterol in einer Dosis von $18 \mu \mathrm{g} 15$ Minuten nach Inhalation gegenüber Plazebo eine optimale Bronchodilatation, die auch durch eine zusätzliche Dosis (kumulativ $27 \mu \mathrm{g}$ ) nicht weiter verbessert werden konnte. Bei Patienten mit schwergradigem Asthma bronchiale war Formoterol als Bedarfsmedikation gegenüber den kurzwirksamen $\beta_{2}$-Sympathomimetika Terbutalin und Salbutamol äquipotent [33]. Zwar steht bei Patienten mit COPD aufgrund der geringeren Variabilität der Beschwerden gegenüber Asthmatikern die kontinuierliche bronchodilatatorische Dauertherapie gegenüber dem Akutgebrauch von $\beta_{2}$-Sympathomimetika im Vordergrund, dennoch könnte die zusätzliche Option eines bedarfsadaptierten Gebrauchs von Formoterol die Therapie der obstruktiven Atemwegserkrankung zumindest vereinfachen [34].

Sowohl in der Formoterolgruppe als auch in der Salmeterolgruppe konnte nach 14 Tagen Dauertherapie in Übereinstimmung mit groß angelegten klinischen Studien zu Asthma und COPD [6,7] eine deutliche Verbesserung der Ausgangslungenfunktion nach entsprechender Karenz gegenüber $\beta_{2}$-Sympathomimetika festgestellt werden. Dabei zeigten sich bezüglich des Ausmaßes der Verbesserung zwischen Formoterol und Salmeterol keine signifikanten Unterschiede. Trotz der verbesserten Ausgangslungenfunktion konnte nach einer erneuten Inhalation von Formoterol bzw. Salmeterol bei allen Patienten in beiden Gruppen eine erneute Verbesserung von sRaw um mindestens 10\% dokumentiert werden.

Die Daten der vorliegenden Untersuchung belegen die gute Verträglichkeit von Formoterol und Salmeterol, die auch in der Langzeittherapie ausreichend dokumentiert worden ist [35-37].

Unerwünschte Nebenwirkungen der Prüfmedikation betrafen die für die Therapie mit $\beta_{2}$-Sympathomimetika typischen $\mathrm{Ne}$ benwirkungen (Kopfschmerzen, Schlafstörungen, Tremor, Schwindel) und wurden etwa gleich häufig wie in anderen Studien [37-39] dokumentiert, wo derartige Ereignisse bei weniger als 5\% der Anwender aufgetreten waren. Das im Rahmen der vorliegenden Studie aufgetretene schwerwiegende, unerwünschte Ereignis wurde vom Prüfarzt nicht mit der Prüfmedikation in Zusammenhang gebracht. Unter der Therapie sowohl mit kurz- als auch langwirksamen $\beta_{2}$-Sympathomimetika sind Hypokaliämien beobachtet worden [35]. In der vorliegenden Untersuchung konnte lediglich bei einem Patienten in der Formoterolgruppe eine Hypokaliämie unter Therapie dokumentiert werden. Eine Tachykardie oder andere Herzrhythmusstörung wurden bei keinem Patienten als unerwünschtes Ereignis dokumentiert. Ebenso blieb die mittlere Herzfrequenz und der mittlere arterielle Blutdruck in beiden Gruppen unter Therapie unverändert. Diese Beobachtungen sind von besonderer Relevanz, da bei einem Großteil der eingeschlossenen Patienten kardiovaskuläre Begleiterkrankungen (Herzinsuffizienz, KHK, Hypertonie) vorlagen. Gerade diese Patienten sollten in besonderem Maße auf evtl. Nebenwirkungen von $\beta_{2}$-Sympathomimetika überwacht werden. Darüber hinaus konnten keine klinisch relevanten Änderungen der übrigen internistischen Laborparameter inklusive der Blutglukose beobachtet werden.

Die vorgestellte Studie weist die üblichen Limitationen einfachblinder, vergleichender Arzneimittelprüfungen auf. Es fehlte ein Kontrollarm (Plazebo) zur differenzierten Bewertung der Ergebnisse. Andererseits fand die Studie unter realistischen Alltagsbedingungen statt, d.h. es wurden auch aktiv rauchende Patienten mit relevanter Ko-Morbidität in die Untersuchung aufgenommen, und auf eine strenge Trennung zwischen Asthma und COPD verzichtet. Das Patientenkollektiv dürfte daher für einen nicht unbeträchtlichen Teil der Patienten mit obstruktiven Atemwegserkrankungen in der täglichen Praxis repräsentativ sein $[40,41]$.

In der vorliegenden Studie konnte erstmalig bei Patienten mit mittel- bis schwergradiger, partiell reversibler Atemwegsobstruktion gezeigt werden, dass Formoterol (Pulverinhalation mittels Aerolizer ${ }^{\circledR}$ ) einen raschen bronchodilatatorischen Effekt auf bodyplethymographische Messgrößen aufweist. Der Wirkeintritt war gegenüber Salmeterol (Pulverinhalation mittels Diskus $^{\circledR}$ ) sowohl initial als auch nach einer 14-tägigen Behandlungsphase deutlich schneller, während die maximale Wirkung für beide Substanzen gleich war. Neben der herkömmlichen Klassifikation von $\beta_{2}$-Sympathomimetika in kurz- und langwirksame sollte zusätzlich zwischen schnell und verzögert wirksamen unterschieden werden.

\section{Literatur}

${ }^{1}$ Naline E, Zhang Y, Qian Yet al. Relaxant effects and durations of action of formoterol and salmeterol on the isolated human bronchus. Eur Respir J 1994; 7: $914-920$

2 Rabe KF, Joerres R, Nowak D et al. Comparison of the effects of Salmeterol and Formoterol on airway tone and responsiveness over 24 hours in bronchial asthma. Am Rev Respir Dis 1993; 147: 1436-1441

${ }^{3}$ Palmqvist M, Persson G, Lazer L et al. Inhaled dry-powder formoterol and salmeterol in asthmatic patients: onset of action, duration of effect and potency. Eur Respir J 1997; 10: $2484-2489$

${ }^{4}$ Van Noord JA, Smeets JJ, Raaijmakers JA et al. Salmeterol versus formoterol in patients with moderately severe asthma: onset and duration of action. Eur Respir J 1996; 9: 1684-1688

${ }^{5}$ Tattersfield AE, Loefdahl CG, Postma DS et al. Comparison of formoterol and terbutaline for as-needed treatment of asthma: a randomised trial. Lancet 2001; 357: 257-261

${ }^{6}$ Pauwels RA, Löfdahl C-G, Postma DS et al. Effect of inhaled Formotero and Budesonide on exacerbations of asthma. N Engl J Med 1997; 337: $1405-1411$

${ }^{7}$ Woolcock A, Lundback B, Ringdal N et al. Comparison of addition of salmeterol to inhaled steroids with doubling of the dose of inhaled steroids. Am J Respir Crit Care Med 1996; 153: 1481 - 1488

${ }^{8}$ National Heart LaBINIoH. Guidelines for the diagnosis and management of asthma. NIH Publication 1998; $98-4051$

${ }^{9}$ Greening AP, Ind PW, Northfield $M$ et al. Added salmeterol versus higher-dose corticosteroid in asthma patients with symptoms on existing inhaled corticosteroid. Lancet 1994; 344: 219-224

${ }^{10}$ Pearlman DS, Liddle R. Controlling asthma symptoms; salmeterol compared with salbutamol in large-scale multi-centre studies. Eur Respir Rev 1994; 21: 301 - 305

${ }_{11}$ Pearlman DS, Chervinsky P, LaForce C et al. A comparison of salmeterol with albuterol in the treatment of mild-to-moderate asthma. N Engl J Med 1992; 327: 1420-1425

${ }^{12}$ Kesten S, Chapman KR, Broder I et al. A three-month comparison of twice daily inhaled formoterol versus four times daily inhaled albuterol in the management of stable asthma. Am Rev Respir Dis 1991; 144: 622-625 
${ }^{13}$ Mahler DA, Donohue JF, Barbee RA et al. Efficacy of salmeterol xinafoate in the treatment of COPD. Chest 1999; 115: 957-965

${ }^{14}$ Manning HL. Bronchdilator therapy in chronic obstructive pulmonary disease. Curr Opin Pulm Med 2000; 6: 99-103

15 Pauwels RA, Buist AS, Calverley PMA et al. Global Strategy for the diagnosis, management, and prevention of chronic obstructive pulmonary disease. NHLBI/WHO Global Initiative for Chronic Obstructive Lung Disease (GOLD) Workshop Summary. Am J Respir Crit Care Med 2001; 163: $1256-1276$

${ }^{16}$ Anthonisen NR, Wright EC. Bronchodilator response in chronic obstructive pulmonary disease. Am Rev Respir Dis 1986; 133: 814 - 819

17 Dow L. Asthma versus chronic obstructive pulmonary disease - exploring why "reversibility versus irreversibility" is no longer an appropriate approach. Clin Exp Allergy 1999; 29: 739- 743

18 Dompeling E, Van Schayck CP, Molema J et al. A comparison of six different ways of expressing the bronchodilating response in asthma and COPD; reproducibility and dependence of prebronchodilator $\mathrm{FEV}_{1}$. Eur Respir J 1992; 5: 975-981

${ }^{19}$ Taube C, Lehnigk B, Paasch K et al. Factor analysis of changes in dyspnea and lung function parameters after bronchodilation in chronic obstructive pulmonary disease. Am J Respir Crit Care Med 2000; 162: $216-220$

${ }^{20}$ Morice AH, Waterhouse JC, Peers EM et al. Use of whole-body plethysmography to compare bronchodilator inhaler efficacy. Respiration 1998; 65: $120-124$

${ }^{21}$ Wallin A, Sandstrom T, Rosenhall L et al. Time course and duration of bronchodilatation with formoterol dry powder in patients with stable asthma. Thorax 1993; 48: 611 -614

22 American Thoracic Society. Standardization of Spirometry-1987 Update. Am Rev Respir Dis 1987; 136: 1285-1298

${ }^{23}$ Petro W, Konietzko N. Lungenfunktionsdiagnostik. In: Ferlinz R, ed. Diagnostik in der Pneumologie. Stuttgart: Georg Thieme Verlag, 1992: 209-281

${ }^{24}$ Klug B, Bisgaard H. Specific airway resistance, interruptor resonance, and respiratory impedance in healthy children aged $2-7$ years. Pediatric Pulmonology 1998; 25: 322 - 331

${ }^{25}$ Barros MJ, Rees PJ. Assessment of reversibility in patients with chronic airflow obstruction. Br J Clin Pract 1992; 46: 132-135

${ }^{26}$ Barnes PJ. Mechanisms in COPD: differences from asthma. Chest 2000; 117: $10-14$
${ }^{27}$ Jans MP, Schellevis FG, van Hensbergen W et al. Management of asthma and COPD patients: feasibility of the application of guidelines in general practice. Int J Qual Health Care 1998; 10: $27-34$

${ }^{28}$ Ryu JH, Scanlon PD. Obstructive lung diseases: COPD, asthma, and many imitators. Mayo Clin Proc 2001; 76: 1144-1153

${ }^{29}$ Cazzola M, Matera MG, Santangelo G et al. Salmeterol and formoterol in partially reversible severe chronic obstructive pulmonary disease: a dose-response study. Respir Med 1995; 89: 357-362

${ }^{30}$ Steffensen I, Faurschou P, Riska H et al. Inhaled formoterol dry powder in the treatment of patients with reversible obstructive airway disease. A 3-month, placebo-controlled comparison of the efficacy and safety of formoterol and salbutamol, followed by a 12-month trial with formoterol. Allergy 1995; 50: 657-663

31 Ullman A, Hedner J, Svedmyr N. Inhaled Salmeterol and Salbutamol in asthmatic patients. Am Rev Respir Dis 1990; 142: 571 - 575

32 Cazzola M, Di Perna F, D'Amato M et al. Formoterol Turbuhaler for asneeded therapy in patients with mild acute exacerbations of COPD. Respir Med 2001; 95: 917-921

${ }^{33}$ Selroos O. Formoterol used as needed - clinical effectiveness. Respir Med 2001; 95: 17-20

${ }^{34}$ Chapman KR, Walker L, Cluley $S$ et al. Improving patient compliance with asthma therapy. Respir Med 2000; 94: 2-9

35 Fuller R. Safety of salmeterol in the treatment of asthma. Eur Respir Rev 1995; 27: $133-137$

${ }^{36}$ Britton MG, Earnshaw JS, Palmer JB. A twelve month comparison of salmeterol with salbutamol in asthmatic patients. Eur Respir J 1992; 5: $1062-1067$

${ }^{37}$ Beeh KM, Wiewrodt R, Salem AE et al. Wirksamkeit und Verträglichkeit der Langzeittherapie mit Salmeterol bei obstruktiven Atemwegserkrankungen. Pneumologie 2000; 54: 225-230

${ }^{38}$ Nathan RA, Seltzer JM, Kemp JP et al. Safety of salmeterol in the maintenance therapy of asthma. Ann Allergy Asthma Immunol 1995; 75: $243-248$

${ }^{39}$ Lundback B, Rawlinson DW, Palmer JB. Twelve months comparison of salmeterol and salbutamol as dry powder formulations in asthmatic patients. Thorax 1993; 48: 148 - 153

40 Beeh KM, Micke P, Ksoll M et al. Cigarette smoking, but not sensitization to Alternaria, is associated with severe asthma in urban patients. J Asthma 2001; 38: 41 - 49

${ }^{41}$ Althuis MD, Sexton M, Prybylski D. Cigarette smoking and asthma symptom severity among adult asthmatics. J Asthma 1999; 36: $257-264$ 